\title{
Design Methodology of Conical Section Shape for Supercavitating Vehicles considering Auto-Oscillation Characteristics
}

\author{
Daijin Li (iD, Fengjie Li, Kan Qin, Chuang Huang ${ }^{D}$, and Kai Luo \\ School of Marine Science and Technology, Northwestern Polytechnical University, Xi'an 710072, China \\ Correspondence should be addressed to Daijin Li; lidaijin@nwpu.edu.cn
}

Received 4 July 2019; Accepted 30 October 2019; Published 10 March 2020

Academic Editor: Lingzhong Guo

Copyright (c) 2020 Daijin Li et al. This is an open access article distributed under the Creative Commons Attribution License, which permits unrestricted use, distribution, and reproduction in any medium, provided the original work is properly cited.

\begin{abstract}
Due to the complexity of the cavity/vehicle and oscillation characteristics, streamlined shape integrated design of conventional fully wetted vehicles is not suitable for supercavitating vehicles. In this paper, a set of design criteria is highlighted to optimize the length and streamlined shape of a conical section subjected to realistic design constraints, which integrate the complex characteristics of the cavity/vehicle system under the condition of auto-oscillation of supercavitating vehicles. The auto-oscillation and its time-domain characteristics are determined. By deriving the equation describing the cavity/vehicle relationship and identifying the maximum amplitude of the Euler angle, the cavity/vehicle tangent point criterion is proposed to determine the theoretical optimum value of the length of the conical section. A method of equal cross-sectional area for gas flow is proposed to design the streamlined shape of the conical section. Water tunnel and autonomous flight experiments were carried out to validate the feasibility of the design methodology developed in this work.
\end{abstract}

\section{Introduction}

Supercavitation is a phenomenon in which a cavity is formed around an underwater vehicle. Since most of the vehicle surface is encompassed by a supercavity, the drag force of the vehicle is significantly reduced compared to conventional fully wetted vehicles, resulting in a ten-fold increase in speed. Supercavitating vehicles (SVs) are composed of a cavitator, a conical section, a cylindrical section, aft vertical rudders, and a nozzle, as shown in Figure 1.

Extensive research on cavitation has been performed for decades. Experimentally, Logvinovich [1] proposed the wellknown principle of independence of cavity sections' expansion, which is regarded as the theoretical basis for modelling supercavities. Many researchers have expanded this theory and advanced its application in SVs. A set of experimental investigations on the mechanisms of supercavitation, ventilated cavitation, hydrodynamic force, and control strategy for SVs was carried out [2-6]. The structural behavior of flexible or stiffened elastic SV s has been studied $[7,8]$. Furthermore, with the development of controllable, maneuvering SVs, some existing research related to supercavitation has also resulted in important advances in the guidance and control problem for SVs [9-12] and the self-noise signatures at the cavitator of SVs [13]. Overall, the previous research on SVs mainly focused on the mechanisms of supercavitation, dynamic modelling, control, etc.

The complexity of the problem is further increased by the development of maneuvering SVs. Various issues must be addressed, such as slope discontinuities in the forces, the effects of time delay, the nominally forward location of the center of pressure, and changes in system parameters as the vehicle executes a maneuver [14]. Among these, the unique characteristics of the cavity/vehicle system comprise the main factor in resolving these issues. The cavity/vehicle system is complex due to the time-delay effect of the supercavity and conic-like self-excited oscillation of the vehicle [15]. The recent literature also identifies outstanding issues associated with the cavity/vehicle system, as well as the inherent instability motion characteristics of SVs. Nguyen et al. [16] examined the system dynamics in terms of nonlinear instabilities and the tail-slap phenomenon, demonstrating that the cavity shape plays a critical role in determining the system dynamics. Lin et al. [17] presented a 


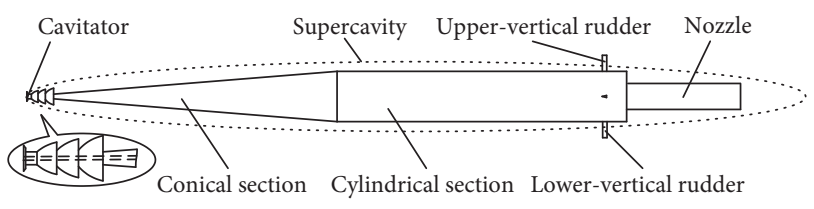

FIgURE 1: Schematic diagram of SV.

dynamic model to interpret the tail-slap phenomenon as a limit cycle motion. Lin et al. [18] studied the bifurcation behavior with respect to a quasistatic variation of the cavitation number, and the tail-slap phenomenon is identified as the consequence of a Hopf bifurcation followed by a grazing event. Considering dominant nonlinearities associated with planing forces, the general characteristics of dynamic behaviors of SVs have been studied by Lv et al. [19] and Xiong et al. [20]. They pointed out that the SV system has complex phenomena, such as coexistence of stable equilibrium point and limit cycle, coexistence of limit cycle and chaotic attractor, and coexistence of multiple limit cycles. The authors of the present work also discovered the intrinsic conic-like oscillation in the motion of SVs [21] and pointed out that conic-like oscillation is attributed to the occurrence of the tail-slap motion in the vertical and horizontal planes and the steady phase difference.

The basic configuration of the vehicle itself is still the major question concerning maneuvering SVs [15]. Limited investigations have been performed on configuration of SVs. In addition, most studies mainly discussed structural behavior, drag characteristics, and cavitator assembly, not mentioning the streamlined shape design principle. Ruzzene [22] did an extensive analysis of both the static and dynamic buckling stabilities of a cylindrical torpedo shell. The effect of varying the number of ring stiffeners was considered in terms of buckling stability. Alyanak et al. [23] presented the optimal structural configuration for a supercavitating torpedo using both radial and longitudinal stiffeners. The optimal stiffener dimensions were presented, along with the optimal number of each kind. However, the overall torpedo dimensions were constant, and the model was a simple cylinder with a conical nose. Furthermore, an algorithm to determine the optimal configuration of the torpedo was presented [24], in which an optimization problem was formulated that determines the general shape of the torpedo in order to satisfy the required performance criteria. Choi et al. [25] used a shape design sensitivity analysis technique (principle of minimum-drag) to determine the optimum shape of the cavitator, carrying out supercavitating flow analysis for a range of cavitation numbers. Uhlman [26] did parametric studies on the supercavitating flow and cavitators for a range of cavitation numbers. Kuklinski [27] also presented experimental studies on the cavitation effect and control performance of different cavitators. The results from the aforementioned two studies show promising agreement. Ahn et al. [15] optimized the configuration and nominal operating speed of an SV by integrating several operational requirements in the optimization process. Kawakami and Arndt [28] experimentally investigated the artificial supercavitation behind a sharp-edged disk for various model configurations. Results regarding supercavity shape, closure, and ventilation requirements versus Froude number were presented. Mokhtarzadeh et al. [29] studied the effect of cavitator shapes on the vehicle stability. They pointed out that the vehicle static stability characteristics depend on the cavitator tangential forces. Park and Rhee [30] researched a turbulent supercavitating flow around a body equipped with a wedge-shaped cavitator, where CFD solutions for the cavity length were consistent with the experimental results.

For integrated design of conventional fully wetted vehicles, the multidisciplinary design optimization method [31], linesearch-based derivative-free approach for nonsmooth constrained optimization [32], collaborative optimization and gradient-free method [33], individual discipline feasible method [34], and other combination design methods to minimize the drag force [35] have been used. In addition, some designed streamlined shapes have been successfully used for expressing the nose and tail sections of fully wetted underwater vehicles, such as Glanville lines [36]. However, it is unlikely that the design of SVs is compatible with current fully wetted vehicles [23]. Due to the complexity of the cavity/vehicle and oscillation characteristics, the streamlined shape design of SVs is also totally different with wetted vehicles.

Focusing on the conical section of SVs, a set of design criteria is presented in this paper to assess the length and streamlined shape of a conical section subjected to realistic design constraints, which integrate the complex characteristics of the cavity/vehicle system under the condition of auto-oscillation of the vehicle. Using the presented design criteria, the optimal length and streamlined shape of a conical section can be sought to maximize the range of stable operation of SVs. The results of water tunnel and autonomous flight experiments verify the feasibility of the proposed design methodology. The main contributions of this paper are as follows. (1) Taking the boundaries of the cavity as the operating constraints, the cavity/vehicle tangent point criterion is proposed to determine the theoretical optimum value of the length of the conical section. (2) A method of equal cross-sectional area for gas flow is proposed to design the streamlined shape of the conical section.

The remainder of this paper is organized as follows. Section 2 expounds the auto-oscillation characteristics of SVs. Section 3 analyzes the time-domain characteristics of SVs, and the conic-like oscillation phenomenon is highlighted. Section 4 proposes the design criteria of the conical section of SVs in detail. Section 5 presents water tunnel and autonomous flight experiments to validate the design method. Finally, several conclusions are drawn in Section 6.

\section{Auto-Oscillation Characteristics of SVs}

To obtain a low-order model that still reflects the essential dynamics in a mathematically tractable form, the following assumptions are made:

(i) The vehicle is sailing with a constant speed.

(ii) The vehicle is sailing on the horizontal plane.

(iii) The rolling angle of the vehicle is zero. 
(iv) The vehicle is being controlled to sail with quite a small yaw angle and sideslip angle. This implies that $\sin \beta \approx \beta, \cos \beta \approx 1, \sin \psi \approx \psi$, and $\cos \psi \approx 1$.

According to the literature [21,37], the kinetic equations based on the assumptions above are presented as follows.

The force balance at the horizontal plane (without control) is described as

$$
m v(\dot{\psi}-\dot{\beta})=-Z .
$$

The moment balance is

$$
\begin{gathered}
J \ddot{\psi}=M_{y}, \\
M_{y}=L_{\alpha} Z .
\end{gathered}
$$

The yaw rate in the ground coordinate system is

$$
\dot{z}_{e}=-v(\psi-\beta) \text {. }
$$

The lateral forces are composed of forces on the cavitator and planing forces. Lateral force can be described in a simplified form:

$$
Z=c_{\alpha} r_{z w}-c_{\omega} \dot{\psi}
$$

where

$$
\begin{aligned}
& r_{z w}=z_{n \tau}-z_{w}, \\
& z_{n \tau}=z_{e \tau}-\psi_{\tau} L_{n}, \\
& z_{w}=z_{e}+\psi L_{w} .
\end{aligned}
$$

The delayed time, $\tau$, is written as

$$
\tau=\frac{L_{t}}{v}
$$

The vehicle length is

$$
L_{t}=L_{n}+L_{w}
$$

The arm of lateral force about mass center is

$$
L_{\alpha}=L_{t}-K_{r \alpha} r_{z w A}-L_{n},
$$

where $r_{z w A}$ is the amplitude of $r_{z w}$, and $r_{z w A}=\sqrt{r_{y}^{2}+r_{z}^{2}}$, describing the deviation between the cavity and the vehicle at the stern plane. Here, $r_{y}$ and $r_{z}$ are the components of the vector from the stern plane center to cavity centerline, as shown in Figure 2, where $O_{b} x_{b} y_{b} z_{b}$ is the body-fixed coordinate system, the expressions of which are given in the Appendix [21, 37].

Equation (11) is the basic hypothesis for the analysis of the motion characteristics of SVs, describing the dependence between the position of force acting point on the shell and the deviation of cavity and vehicle at the tail.

Considering equation (1), the derivative of equation (4) with respect to time is

$$
m \ddot{z}_{e}=Z .
$$

According to equations (2) and (3), the moment balance can be rewritten as

$$
J \ddot{\psi}=L_{\alpha} Z .
$$

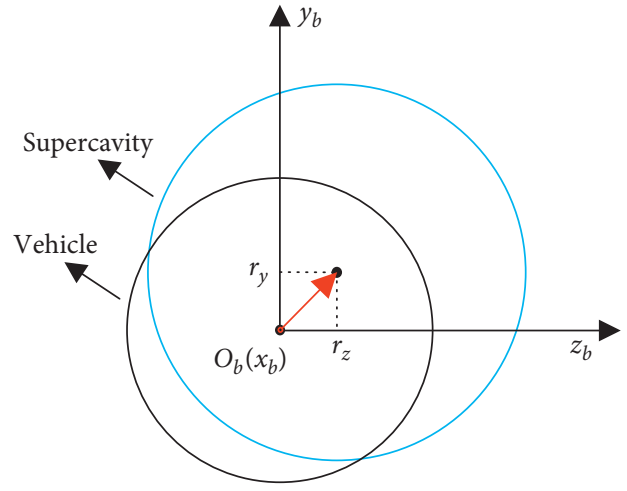

FIGURE 2: Relationship between vehicle and cavity at stern plane (back view).

Using equations (12) and (13) yields

$$
L_{\alpha} m \ddot{z}_{e}=J \ddot{\psi} \text {. }
$$

Equations (12)-(14) express the basic dynamic relationship of SVs.

Equations (1) and (13) yield

$$
\dot{\beta}=\frac{J}{m v L_{\alpha}} \ddot{\psi}+\dot{\psi} \text {. }
$$

Its transfer function is

$$
\frac{\ell(\beta)}{\ell(\psi)}=\left(1+s \frac{J}{m v L_{\alpha}}\right),
$$

where $\ell$ denotes the Laplace transform. According to equation (13), we obtain

$$
\frac{\ell(\psi)}{\ell(Z)}=\frac{L_{\alpha}}{s^{2} J^{j}}
$$

Based on equations (5)-(8), we obtain

$$
\frac{Z}{c_{\alpha}}=\left(z_{e \tau}-z_{e}-\psi_{\tau} L_{n}-\psi L_{w}\right)-\frac{c_{\omega}}{c_{\alpha}} \dot{\psi} .
$$

Incorporated with time delay, sideslip angle at time $t$ can be approximated as

$$
\psi \approx \psi_{\tau}+\frac{\tau\left(\dot{\psi}+\dot{\psi}_{\tau}\right)}{2} .
$$

Its transfer function is

$$
\frac{\ell\left(\psi_{\tau}\right)}{\ell(\psi)} \approx \frac{1-(\tau) /(2) s}{1+(\tau) /(2) s} .
$$

Similarly, the following relationship can be obtained:

$$
\frac{\ell\left(z_{e \tau}\right)-\ell\left(z_{e}\right)}{\ell\left(z_{e}\right)} \approx \frac{-\tau s}{1+(\tau) /(2) s} .
$$

From equations (4), (18), (20), and (21), we obtain

$$
\begin{aligned}
-\frac{\ell(Z)}{c_{\alpha}}= & \frac{v \tau}{1+(\tau) /(2) s} \ell(\beta) \\
& +\left(\frac{-v \tau}{1+(\tau) /(2) s}+\frac{1-(\tau) /(2) s}{1+(\tau) /(2) s} L_{n}+L_{w}+\frac{c_{\omega}}{c_{\alpha}} s\right) \ell(\psi) .
\end{aligned}
$$


Using equations (9), (16), and (22) yields

$-\frac{\ell(Z)}{\ell(\psi) c_{\alpha}}=s \frac{c_{\omega}}{c_{\alpha}}+\left(L_{w}-L_{n}+\frac{2 J}{m L_{\alpha}}\right)+\frac{2\left(L_{n}-\left(J / m L_{\alpha}\right)\right)}{((\tau) /(2) s+1)}$.

Now, we assume the following relationship is true:

$$
L_{\alpha}=\frac{J}{m L_{n}} \text {. }
$$

Further, using equation (10) and the relationship of $c_{\omega} \ll c_{\alpha}$, equation (23) can be approximately simplified as

$$
\frac{\ell(Z)}{\ell(\psi)}=-c_{\alpha} L_{t} \text {. }
$$

Similarly, if the relationship expressed in equation (24) is assumed to be true, equation (17) can also be simplified as

$$
\frac{\ell(\psi)}{\ell(Z)}=\frac{1}{s^{2} m L_{n}}
$$

We can see that in equations (25) and (26), the phase relation of the variables $Z$ and $\psi$ satisfies the existence condition of the auto-oscillation. The following relationship can be obtained:

$$
s^{2} \ell(\psi)=\frac{-c_{\alpha} L_{t}}{m L_{n}} \ell(\psi)
$$

If equation (27) is true, the angular frequency of the auto-oscillation is

$$
\omega=\sqrt{\frac{L_{t} c_{\alpha}}{L_{n} m}}
$$

According to the analysis above, the auto-oscillation can be generated, under the sufficient conditions of equations (24) and (28).

\section{Time-Domain Analysis of Auto-Oscillation}

Because the mathematical derivation of the auto-oscillation is based on the approximate transfer relationship of equations (20) and (21), further analysis in the time-domain is necessary.

Assuming $\psi$ is a simple harmonic oscillation, we obtain

$$
\psi=\psi_{A} \cos \omega t .
$$

Further considering equation (14), we obtain

$$
z_{e}=\frac{J}{m L_{\alpha}} \psi
$$

Based on equations (29), (30), (7), and (8), the following equations can be obtained:

$$
\begin{aligned}
z_{n \tau} & =\left(\frac{J}{m L_{\alpha}}-L_{n}\right) \psi_{A} \cos (\omega t-\omega \tau), \\
z_{w} & =\left(\frac{J}{m L_{\alpha}}+L_{w}\right) \psi_{A} \cos \omega t .
\end{aligned}
$$

Using equations (31), (32), and (6) yields

$$
r_{z w}=\left(\frac{J}{m L_{\alpha}}-L_{n}\right) \psi_{A} \cos (\omega t-\omega \tau)-\left(\frac{J}{m L_{\alpha}}+L_{w}\right) \psi_{A} \cos \omega t
$$

Note that based on equations (5) and (13), $r_{z w}$ can also be obtained:

$$
r_{z w}=-\psi_{A}\left(\frac{\omega c_{\omega}}{c_{\alpha}} \sin \omega t+\frac{\omega^{2} J}{c_{\alpha} L_{\alpha}} \cos \omega t\right) .
$$

Comparing equations (33) and (34), the following equation can be obtained:

$$
\bar{C} \cos \omega t=\bar{S} \sin \omega t .
$$

The expressions of the coefficients $\bar{C}$ and $\bar{S}$ are given in the Appendix.

If equation (35) is true, the following conditions should be met:

$$
\left\{\begin{array}{l}
\bar{S}=0 \\
\bar{C}=0 .
\end{array}\right.
$$

Equation (36) can be transformed into

$$
L_{\alpha}=\frac{J}{m L_{n}\left(1-\left(c_{\omega} \omega / c_{\alpha} L_{n} \sin \omega \tau\right)\right)} .
$$

Combining equations (36) and (37), we obtain

$$
\frac{\omega^{3} m c_{\omega}}{c_{\alpha}^{2} \sin \omega \tau}-\omega^{2} \frac{m L_{n}}{c_{\alpha}}+\omega \frac{c_{\omega}(\cos \omega \tau-1)}{c_{\alpha} \sin \omega \tau}+L_{t}=0 .
$$

Solving equation (38) by the trial-and-error method and using equation (28) as the initial value, angular frequency, $\omega$, can be obtained. In addition, $L_{\alpha}$ can be obtained by equation (37).

Furthermore, according to equation (11), we obtain

$$
r_{z w A}=\frac{L_{t}-L_{n}-L_{\alpha}}{K_{r \alpha}} .
$$

Combined with equation (34), the amplitude of the autooscillation can be obtained as follows:

$$
\psi_{A}=\frac{r_{z w A}}{(\omega) /\left(c_{\alpha}\right) \sqrt{c_{\omega}^{2}+\left((\omega J) /\left(L_{\alpha}\right)\right)^{2}}} .
$$

Now, we consider the following relationship:

$$
c_{\alpha} r_{z w} \approx \frac{1}{2} \rho S v^{2} c_{z}^{\beta} \frac{r_{z w}}{L_{t}} \text {. }
$$
[38]

Moreover, the relative density of the vehicle is defined as

$$
\mu=\frac{2 m}{\rho S L_{t}} \text {. }
$$

The angular frequency of the auto-oscillation shown as equation (28) can be simplified to

$$
\omega=v \sqrt{\frac{c_{z}^{\beta}}{\mu L_{n} L_{t}}} .
$$


Thus, it can be seen that the frequency of auto-oscillation is proportional to the sailing speed, inversely proportional to the physical dimension, proportional to the square root of the position derivative of the lateral force coefficient, and is closely related to the location of the mass center.

Based on equation (16), the sideslip angle is described as

$$
\beta=\beta_{A} \cos \left(\omega t+\varphi_{\psi}\right)
$$

where

$$
\begin{aligned}
\beta_{A} & =\frac{\psi_{A}}{K_{\psi}}, \\
K_{\psi} & =\frac{1}{\sqrt{\left((J \omega) /\left(L_{\alpha} m v\right)\right)^{2}+1}}, \\
\cos \varphi_{\psi} & =K_{\psi}, \\
\sin \varphi_{\psi} & =\frac{J \omega}{L_{\alpha} m v} K_{\psi},
\end{aligned}
$$

where $K_{\psi}<1$ and $\varphi_{\psi}$ is in the first quadrant.

According to the definition of trajectory deflection angle, we obtain

$$
\Psi=\psi-\beta
$$

Combined with equation (4), this yields

$$
\dot{z}_{e}=-v \Psi
$$

Again, combined with equation (30), the trajectory deflection angle is described as

$$
\Psi=\psi_{A} \frac{J \omega}{L_{\alpha} m v} \sin \omega t .
$$

Using equation (7) and assuming the amplitude is zero, we obtain

$$
0=z_{e}-\psi L_{n}^{\prime}
$$

where $L_{n}^{\prime}$ is the distance from the instantaneous velocity center (where the amplitude is zero) to the mass center, which can be obtained by combining equations (30) and (37).

$$
L_{n}^{\prime}=\frac{J}{m L_{\alpha}}=L_{n}\left(1-\frac{c_{\omega}}{c_{\alpha}} \frac{\omega}{L_{n} \sin \omega \tau}\right) .
$$

Distance from the instantaneous velocity center to the cavitator can be calculated as follows:

$$
x_{s x}=L_{n}-L_{n}^{\prime}=\frac{c_{\omega}}{c_{\alpha}} \frac{\omega}{\sin \omega \tau} L_{n}
$$

This means that the distance from the instantaneous velocity center to the cavitator is very small due to the relationship of $c_{\omega} \ll c_{\alpha}$.

The oscillation position at any position on the vehicle is described as

$$
z_{x}=\left(x-x_{s x}\right) \psi
$$

where $x$ originates at the cavitator and is along the symmetry axis of the vehicle.

Furthermore, the oscillation position at the vertex center of the cavitator is

$$
z_{x n}=-x_{s x} \psi
$$

According to the principle of independence of the cavity sections expansion by Logvinovich [1], the symmetry axis of the supercavity appears as a spiral based on the analysis above, which is also consistent with the experimental photograph in Figure 3. The spiral pitch is described as

$$
S_{c}=\frac{2 \pi v}{\omega}
$$

To summarize, the SVs move in the form of a spatial conic-like auto-oscillation, which can be described as in Figure 4. Projected onto a plane, the spatial oscillation is shown as the well-known "tail-slap" phenomenon, as shown in Figure 5.

\section{Design Criteria of Conical Section}

4.1. Length of Conical Section. The shape of the cavity affects the dynamics of the vehicle and cavity. The description of the cavity closure zone is the most challenging issue in describing cavity shape. According to the known theoretical closure schemes, the cavity in this study most closely resembles the Ryabushinsky scheme [5], in which the cavity is closed on the aft hull surface. Several models exist for predicting the size and shape of the cavity under various flow conditions. Even for the simple case of steady flow, many different models have been proposed. For investigating the basic relationship between the supercavity and the vehicle, the elegant semiempirical relations of Logvinovich [1] (in cases where the cavitation number is small and the gravity influence is negligible) are convenient for predicting the cavity as follows:

$$
\begin{aligned}
R_{c \max } & =R_{n} \sqrt{\frac{c_{x n}}{k_{1} \sigma}}, \\
L_{c \max } & =2 R_{n} k_{2} \frac{\sqrt{c_{x n}}}{\sigma}, \\
R_{c} & =R_{c \max } \sqrt{1-\left(1-\frac{R_{n}^{2}}{R_{c \max }^{2}}\right)\left(1-\frac{2 x}{L_{c \max }}\right)^{2}}, \\
k_{2} & \approx \sqrt{-\ln \sigma} .
\end{aligned}
$$

The expected shape of the supercavity is a slender, nearly axisymmetric cavity, closing at the afterbody of the vehicle, as shown in Figure 6 . At the cavity closure point, $x=L_{t}$ and $R_{c}=R_{t}$ are valid.

Combining equations (55)-(57) yields

$$
A_{\sigma} \sigma^{2}-B_{\sigma} \sigma+C_{\sigma}=0
$$

The expressions of the coefficients $A_{\sigma}, B_{\sigma}$, and $C_{\sigma}$ are given in the Appendix. 


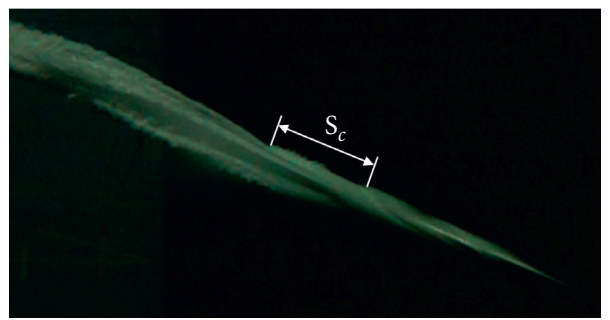

Figure 3: An experimental photograph of a SV.

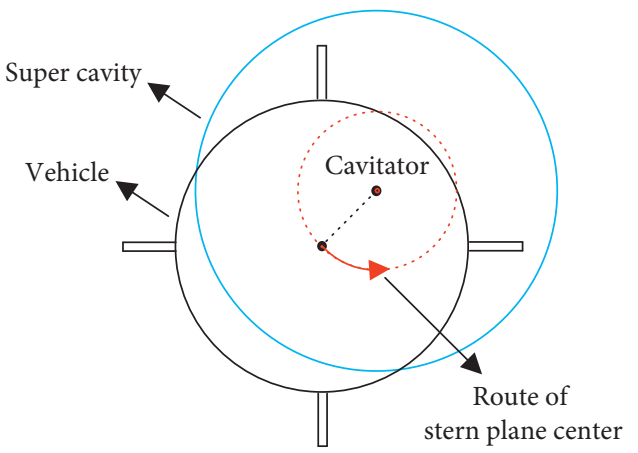

Figure 4: Auto-oscillation of SVs (back view).

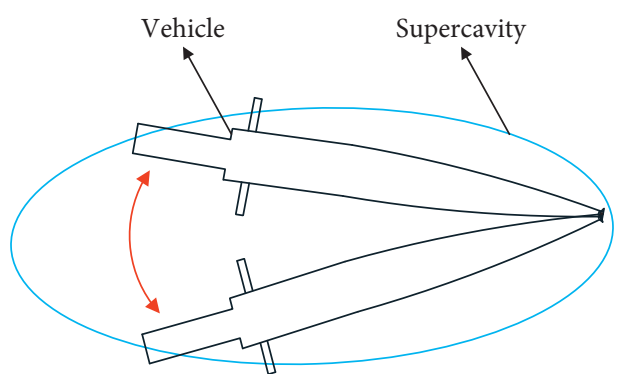

FIgURE 5: Tail-slap phenomenon of SVs.

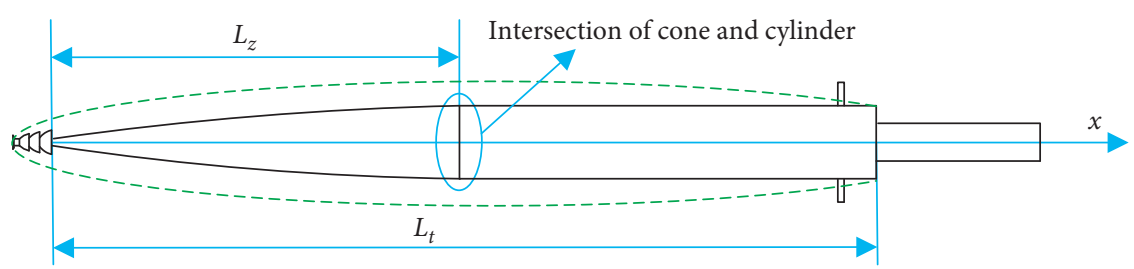

FIgURE 6: The expected shape of the supercavity.

The meaningful root of equation (59) is obtained as

$$
\sigma=\frac{B_{\sigma}-\sqrt{B_{\sigma}^{2}-4 A_{\sigma} C_{\sigma}}}{2 A_{\sigma}}
$$

where the constraint relationship between cavitator radius and cavitation number is identified.

Here, the design principle of cavity closure place is addressed. Due to the existence of the spatial conic-like autooscillation, the hull transiently planes the cavity, where partial cavitation is formed. It seems that the cavity is much smaller than in the nominal design condition. This partial cavitation is even more serious in the case of the vehicle executing a maneuver. With the intensifying of the oscillation, the cavity closure zone is actually driven forward along the body, causing the cavity to close farther upstream. It even moves to the conical section of the vehicle, as shown in Figure 7. In this state, the drag of the vehicle is increased significantly. Thus, the shape parameters of conical section should be optimized so that the cavity closure zone can be located at the expected position even in the case of oscillation. 


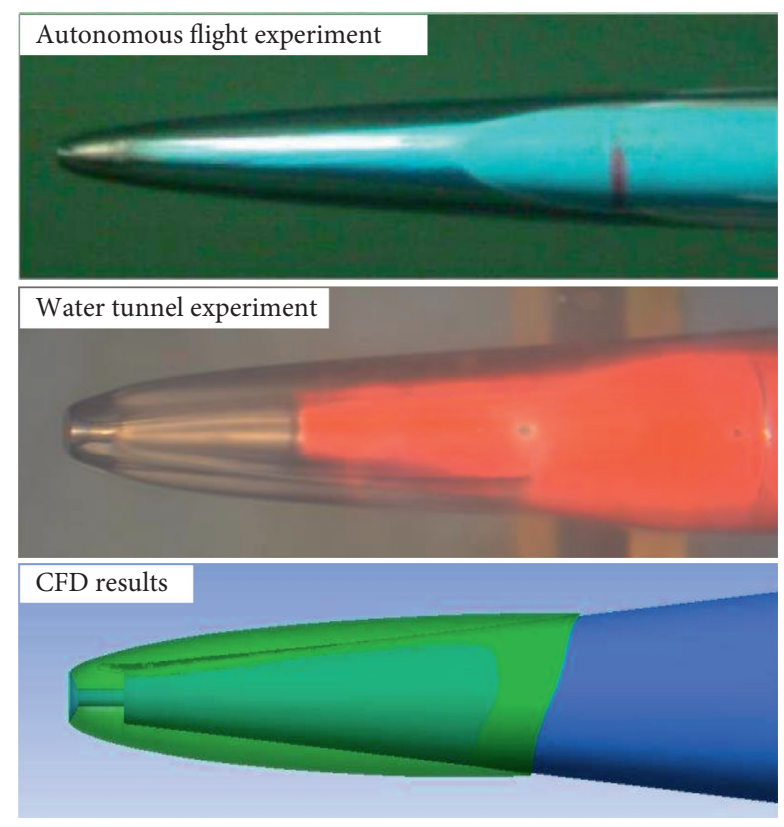

Figure 7: Partial cavitation (closes at conical section).

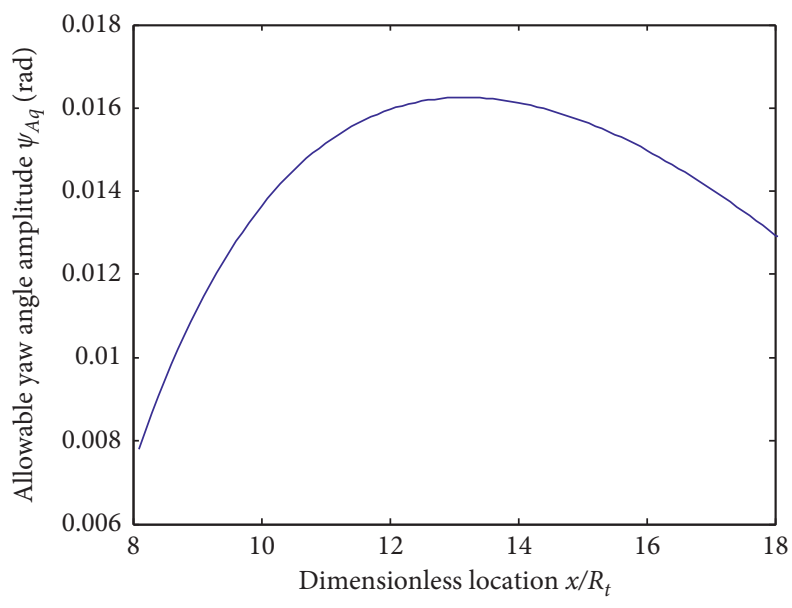

FIGURE 8: Relationship between allowable yaw angle amplitude and length of conical section.

TABLE 1: Cavity/vehicle tangent point criteria of conical section length.

\begin{tabular}{lcc}
\hline Design criterion & Conical section length $L_{z}$ & Relationship of cavity and body \\
\hline$\psi_{\text {Aq } \max }<\psi_{A}$ & Arbitrary $L_{z}$ & Cavity closes at cone \\
$\psi_{\text {Aq } \max }=\psi_{A}$ & $L_{z}=x_{\max }$ & Cavity tangent to vehicle surface at intersection of cone and cylinder \\
$\psi_{\text {Aq } \max }>\psi_{A}$ & $L_{z} \leq x_{\max }$ & \\
\hline
\end{tabular}

Taking the yaw channel of the vehicle as an example and according to equations (52) and (29), the relative position relationship between the supercavity and the vehicle is

$$
\begin{aligned}
& \left(z_{n \tau}+R_{c}\right)-\left(z_{x}+R_{t}\right)=R_{c}-R_{t} \\
& -\psi_{A}\left\{\left[x_{s x}\left(\cos \omega \tau_{x}-1\right)+x\right] \cos \omega t+x_{s x} \sin \omega \tau_{x} \sin \omega t\right\},
\end{aligned}
$$

where the delayed time, $\tau_{x}$, is written as

$$
\tau_{x}=\frac{x}{v} \text {. }
$$

Assuming amplitude $\psi_{A}$ in equation (61) is zero, the supercavity profile is tangent to the hull of the vehicle. That is, at any axial position $x$, there exists only one allowable yaw angle amplitude, $\psi_{A q}$, making the supercavity tangent to the surface of the cylindrical section. 


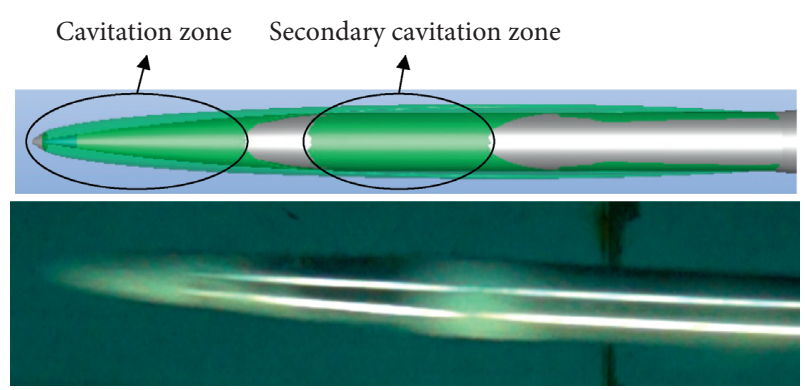

Figure 9: Secondary cavitation from CFD and experiments.

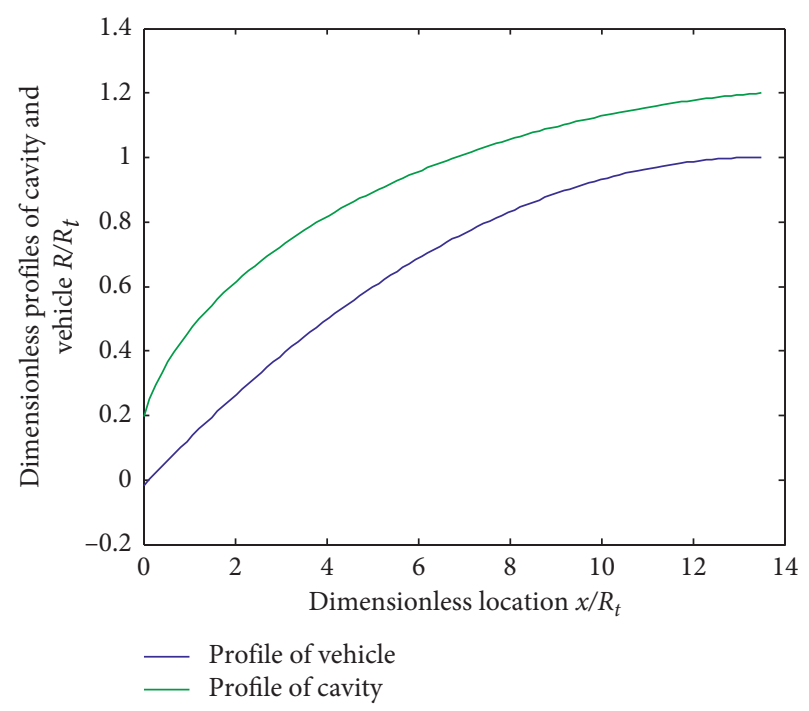

Figure 10: Profile of conical section.

$$
\psi_{A q}=\frac{R_{c}-R_{t}}{\sqrt{\left[x_{s x}\left(\cos \omega \tau_{x}-1\right)+x\right]^{2}+\left(x_{s x} \sin \omega \tau_{x}\right)^{2}}} .
$$

Based on the design experience, assuming the aspect ratio of the vehicle is 12 , the radius of the disk cavitator is $0.2 R_{t}$, and the supercavity closes at the afterbody of the vehicle, the relationship between allowable yaw angle amplitude and length of conical section can be obtained as shown in Figure 8.

From Figure 8, there exists one theoretical optimum value, $x_{\max }$, which supports the maximum amplitude of the yaw angle, $\psi_{\text {Aq max }}$, because the functional relationship described by equation (63) is a convex, decreasing parabolic curve.

If the actual yaw angle amplitude, $\psi_{A}$, obtained from equation (40) is exactly equal to the maximum amplitude $\psi_{A q \text { max }}$, the optimum value of $x_{\max }$, corresponding to $\psi_{\text {Aq max }}$, can be identified as the intersection of the conical section and the cylindrical section. The length of the conical section is identified as $L_{z}=x_{\max }$.

If $\psi_{A q \max }<\psi_{A}$, the cavity closes at the conical section for the arbitrary $L_{z}$.

If $\psi_{A q \max }>\psi_{A}$, the cavity closes at the conical section when $L_{z} \leq x_{\max }$.

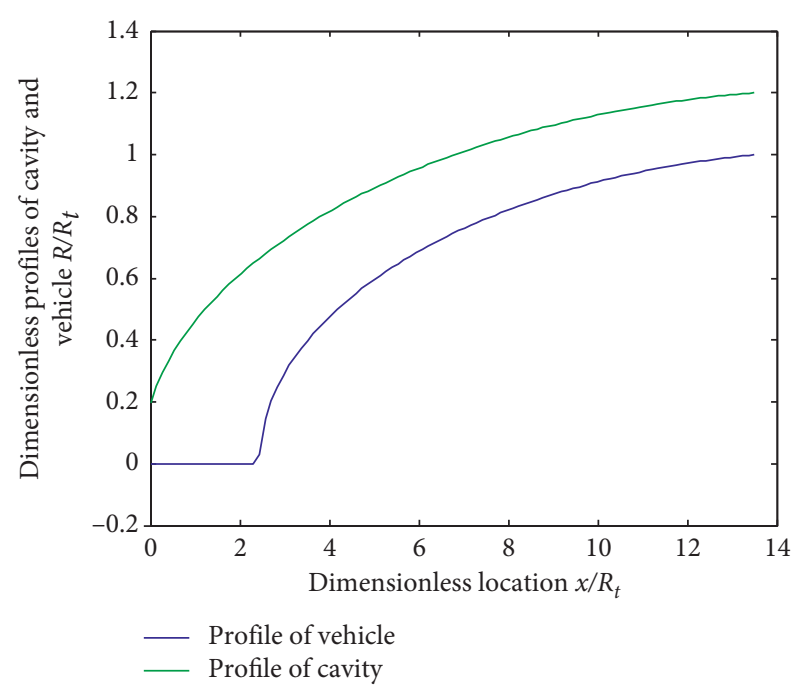

FIgURE 11: Profile of conical section for ventilated SVs.

TABle 2: Parameters in water tunnel experiments.

\begin{tabular}{lccccc}
\hline$R_{n}$ & $v_{\infty}$ & $P_{\infty}$ & Fr & $\sigma_{c}$ & $\sigma_{v}$ \\
\hline $5 \mathrm{~mm}$ & $8 \mathrm{~m} / \mathrm{s}$ & $70 \mathrm{kPa}$ & 25.54 & 0.08 & 1.4865 \\
\hline
\end{tabular}

The above criteria for the length of the conical section, which are named "cavity/vehicle tangent point criteria" in this work, are summarized in Table 1.

Actually, during the sailing of SVs, there is an intersection angle between the cavity centerline and the vehicle centerline because of the existing vehicle attack angle and the buoyancy forces on the cavity. With the intersection angle increased, the cavity closure zone on the one side of the body is driven forward along the body, which causes the increasing drag and lift, referred to as planing force. The acting point of this planing force calculated from equation (37) is also driven forward.

When the action point is behind the mass center, the planing moment is a restoring moment. When the action point moves to the front of the mass center, the planing moment is an overturning moment, and the motion stability of the vehicle is significantly reduced. By adjusting the mass distributions, the larger arm of planing force can be obtained, which can maximize allowable range for the motion stability of the vehicle.

Conversely, if the cavity is not tangent to the surface of vehicle but closes at the conical section, there will be a pressure drop in the wake of the intersection of cone and the cylinder because of the strong bending of liquid streamlines. In such a situation, "secondary cavitation" even appears, as shown in Figure 9.

4.2. Streamlined Shape of Conical Section. For the ventilated SVs, an annular ventilation passage is formed between the conical section profile and the supercavity wall. To stabilize the ventilated gas flow and avoid "impact" on the cavity wall, the cross-sectional area of gas flow (ventilation area) can be designed to be constant. Here, equal cross-sectional area for 


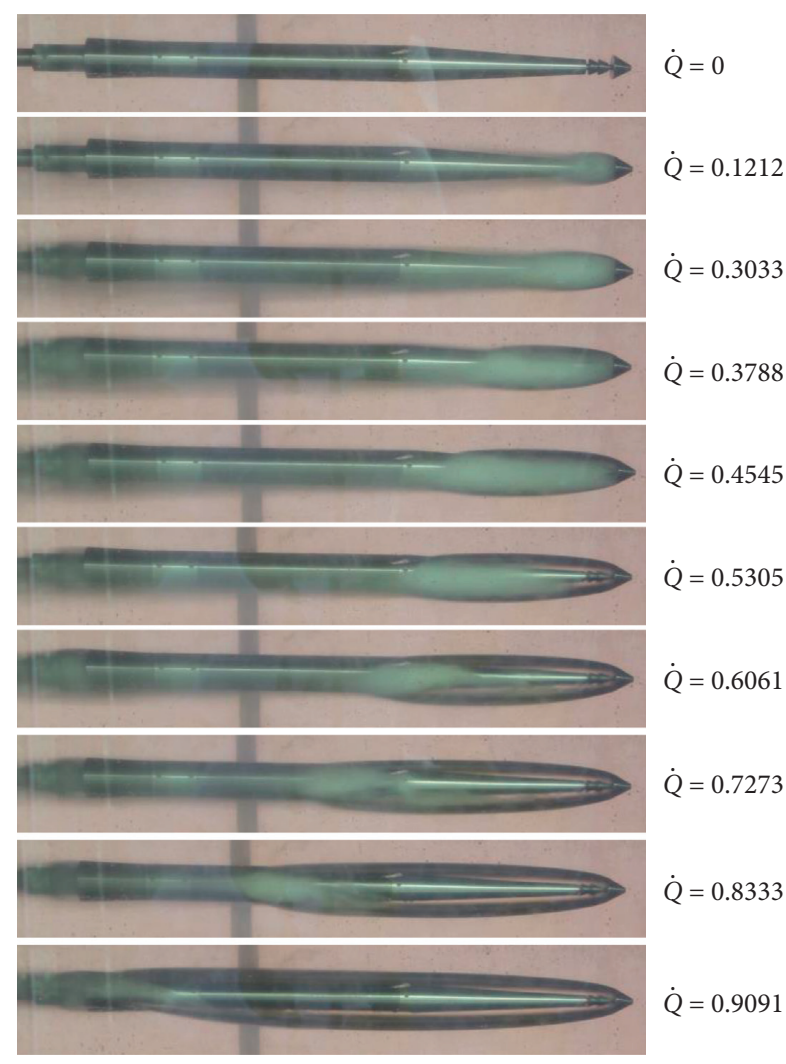

Figure 12: Photographs of ventilated cavities at water tunnel.

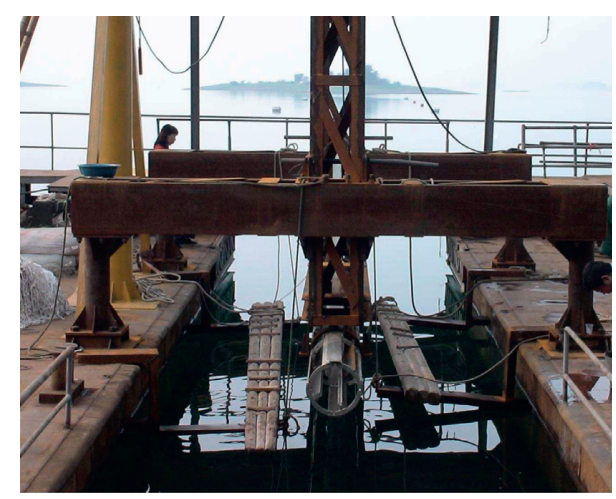

Figure 13: Lakebed autonomous flight experimental model.

gas flow is the design criteria for the streamlined shape of conical section. In this case, the velocity of gas flow is approximately constant.

From equation (63), the position where the cavity is tangent to the vehicle surface can be obtained, where the square difference between the radius of the supercavity and the vehicle is the ventilation area. Assuming that the ventilation area is constant, and incorporated with equations (55)-(57), the following relation can be obtained:

$$
\frac{R_{n}^{2} c_{x n} C_{1}\left(C_{2 q}^{2}-C_{2}^{2}\right)}{k_{1} \sigma}=R_{z}^{2}-R_{t}^{2} .
$$

The expressions of the coefficients $C_{2 q}, C_{1}$, and $C_{2}$ are given in the Appendix.

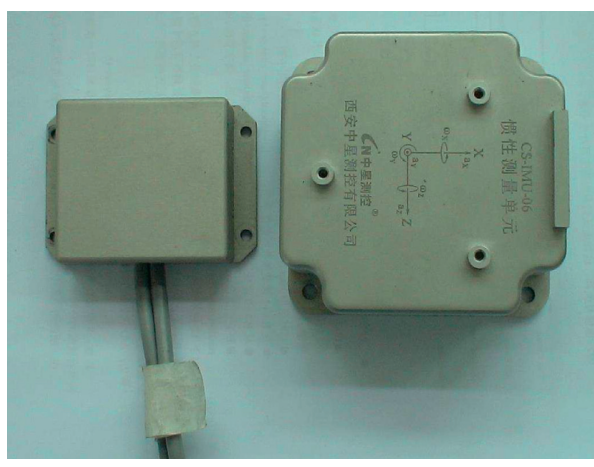

FIGURE 14: Inertial measurement unit.

Equation (64) can be transformed into

$$
R_{z}=\sqrt{\frac{c_{x n} R_{n}^{2} C_{1}\left(C_{2 q}^{2}-C_{2}^{2}\right)}{k_{1} \sigma}+R_{t}^{2}} .
$$

Based on equation (65), the profile of the conical section is shown in Figure 10. For a ventilated SV, ventilated devices are fixed in the front of the conical section, with the length usually less than $2.5 R_{t}$ for a small-caliber $\mathrm{SV}$, as shown in Figure 11.

\section{Experiments for Streamlined Shape of Conical Section}

Using the method given above, an SV model for water tunnel experiments with a cylindrical section diameter of $40 \mathrm{~mm}$ and conical cavitator bottom diameter of $10 \mathrm{~mm}$ was designed. Experiments for the supercavity shape property were carried out with the ventilation method at the highspeed water tunnel of Northwestern Polytechnical University in China.

Fundamental similarity parameters for ventilated supercavities are the cavitation number and Froude number:

$$
\begin{gathered}
\sigma_{c}=\frac{P_{\infty}-P_{c}}{0.5 \rho v_{\infty}^{2}}, \\
\mathrm{Fr}=\frac{v_{\infty}}{\sqrt{2 g R_{n}}} .
\end{gathered}
$$

The natural cavitation number is defined as

$$
\sigma_{v}=\frac{P_{\infty}-P_{v}}{0.5 \rho v_{\infty}^{2}} .
$$

In the case of an axisymmetric cavitator, the dimensionless coefficient of the rate is

$$
\overline{\mathrm{Q}}=\frac{\dot{\mathrm{Q}}}{4 v_{\infty} R_{n}^{2}} .
$$

In the experiment, velocity and pressure of the mainstream are first fixed as constant. Series cavitation numbers can be obtained by operating the flow rate and pressure of the ventilation gas. Different shapes of cavities are formed. Here, the parameters in the water tunnel experiments are 
TABLE 3: Parameters in autonomous flight experiments.

\begin{tabular}{lcccccc}
\hline Vehicle radius & Vehicle length & Vehicle Mass & Depth & Cruising speed & Thrust & Sailing time \\
\hline $101.5 \mathrm{~mm}$ & $2500 \mathrm{~mm}$ & $100 \mathrm{~kg}$ & $3 \mathrm{~m}$ & $204 \mathrm{kN}$ & $25 \mathrm{kN}$ & $4.1 \mathrm{~s}$ \\
\hline
\end{tabular}

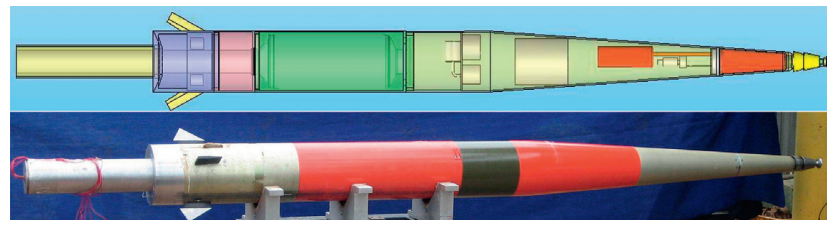

FIGURE 15: Lakebed autonomous flight experimental model.

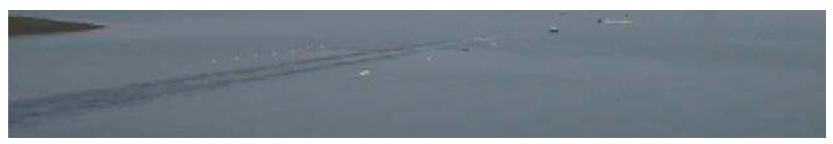

FIgURE 16: Surface trajectory.

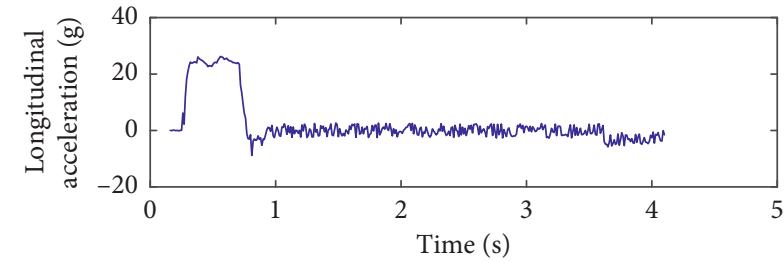

(a)

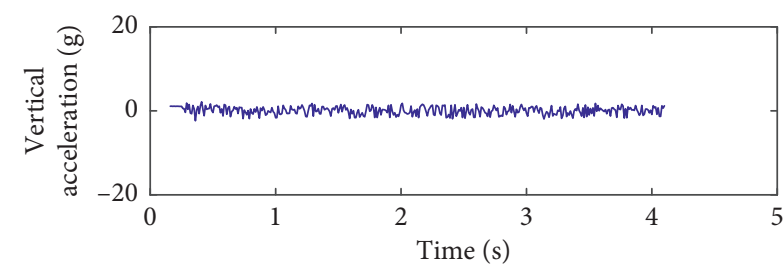

(c)

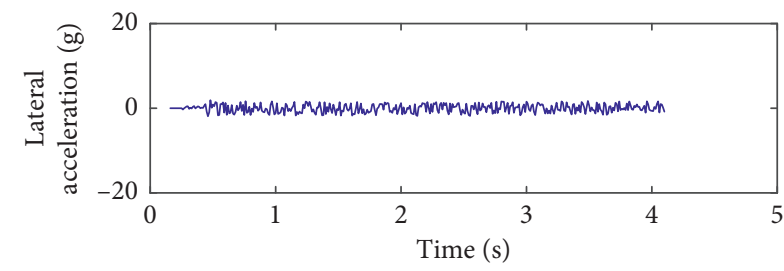

(e)

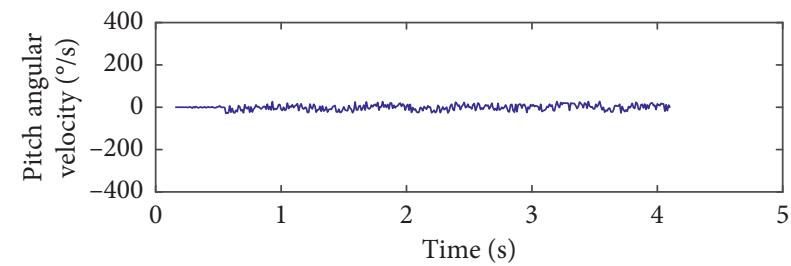

(b)

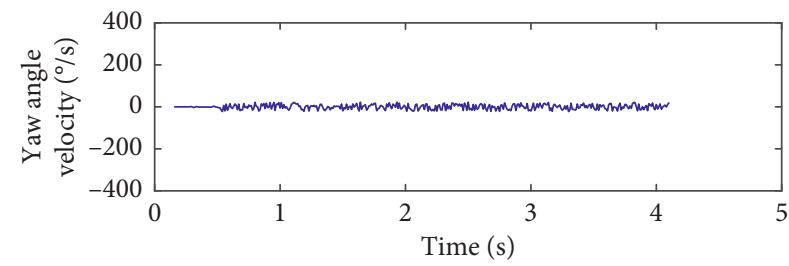

(d)

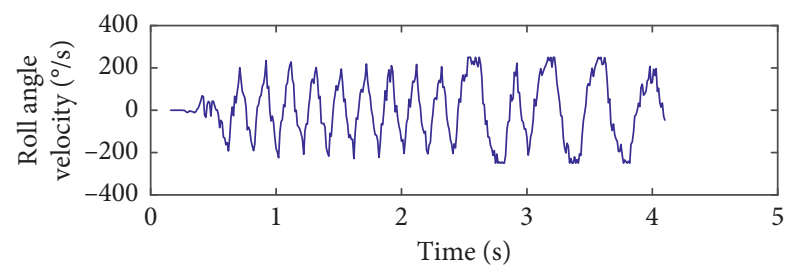

(f)

FIGURE 17: Raw data from the autonomous flight experiment.

shown in Table 2. The volumetric flow rate of the gas increases from bottom to top $\left(0.9091 \mathrm{~m}^{3} / \mathrm{s}\right)$.

Figure 12 shows photographs of ventilated cavities at the same cavitation number and a different volumetric flow rate of gas.

According to the results, when the gas flow rate is less than about 0.5305 , the cavity closes at the conical section, and partial cavitation is formed. When the gas flow rate is about 0.6061 , the cavity is tangent to the vehicle surface at the intersection of the cone and cylinder. If the gas flow rate continues to increase, the cavity can smoothly cross the intersection of the cone and cylinder and then close at the cylindrical section. When the gas flow rate reaches to 0.9091 , the cavity closes at the tail and most of the surface is enveloped by the continuous cavity. The tail of the cavity axis deforms upward due to gravity.

The experimental results show that the designed conical shape of the vehicle can realize the expected supercavitation. However, the auto-oscillation characteristics of SVs are difficult to simulate in the water tunnel laboratory because 


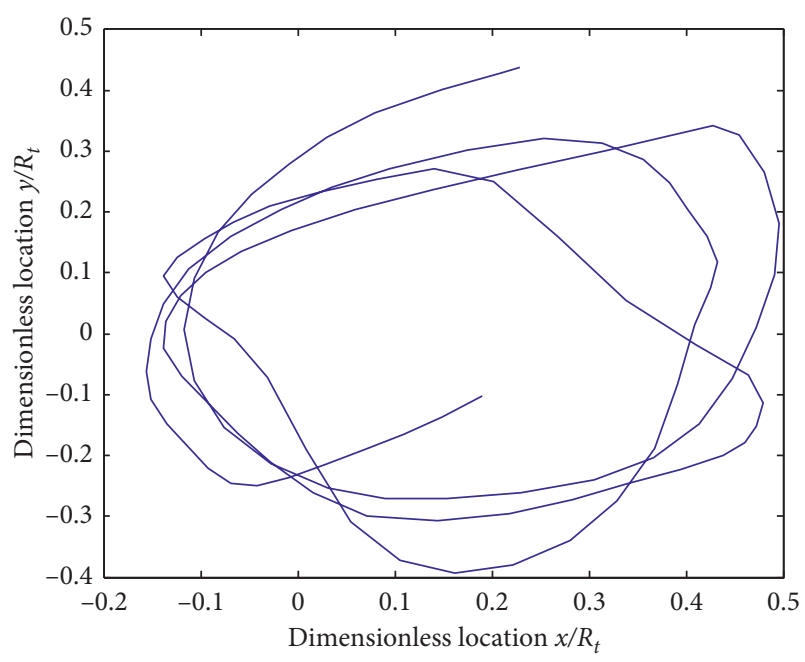

FIgURE 18: Track of tail center of SV.

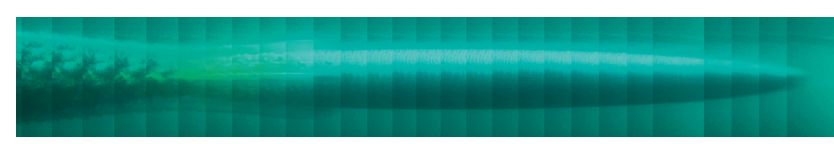

FIGURE 19: Supercavity profile after image mosaic.

the existence of struts restricts the movement of the model. Further autonomous flight experiments can be carried out for a more comprehensive verification.

Furthermore, to validate the design method presented here, lakebed autonomous flight experiments were carried out. In the autonomous flight experiments, the SV was launched from a stockade launch tube, which is mounted on a pontoon catamaran. The launch platform is shown in Figure 13. An inertial measurement unit was used to measure the triaxial acceleration and angular velocity, as shown in Figure 14. A PC104 was used as the central control unit to perform the sailing control and for storage of test data. An underwater high-speed camera was fixed near the launch pad to catch the supercavity profile. The parameters in the autonomous flight experiments are shown in Table 3. The designed experimental SV is shown in Figure 15. Figure 16 shows the straight flight surface trajectory of the experimental model.

The time history of the raw data from the inertial measurement unit is shown in Figure 17. The track of the stern plane center can be obtained by multiplying the tangential components of the time-varying angles, as shown in Figure 18, which can reflect the auto-oscillation of the vehicle in the autonomous flight experiment.

Ventilation devices with gas from gunpowder were used. The mass flow rate of the ventilation gas was about $100 \mathrm{~g} / \mathrm{s}$ at the cruising speed. The temperature of the gas was about $800 \mathrm{~K}$. Figure 19 shows photographs of the ventilated supercavities. The cavity is continuous and can completely envelop the surface of the SV. In particular, the cavities transit smoothly at the intersection of cone and cylinder, and there is no "secondary cavitation," as shown in Figure 20. The results of the autonomous flight experiments verified the feasibility of the proposed design method.

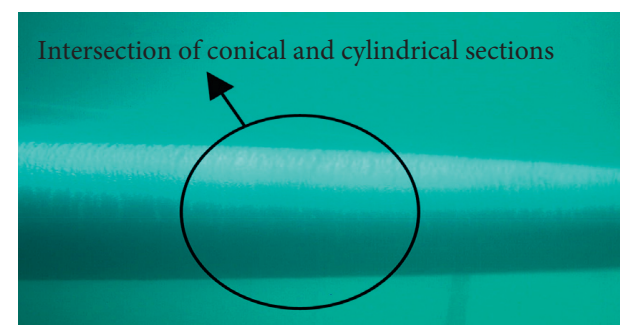

FIGURE 20: Single-frame photograph of supercavity profile.

\section{Conclusions}

In this paper, a set of design criteria is presented to assess the length and streamlined shape of a conical section subjected to realistic design constraints, which integrate the complex characteristics of the cavity/vehicle system under the condition of auto-oscillation of supercavitating vehicles. Key findings are as follows:

(1) According to equation (43), the auto-oscillation frequency of SVs is proportional to sailing speed, inversely proportional to physical dimension, proportional to the square root of the position derivative of the lateral force coefficient, and closely related to the location of the mass center.

(2) In the case of auto-oscillation, the length of the conical section can be designed by identifying the maximum amplitude of the Euler angle. The stability of the SVs is closely related to the position of the mass center. The larger arm of planing force can provide a larger allowable range for the motion stability.

(3) The streamlined shape of the conical section can be designed using the method of equal cross-sectional area to stabilize the gas flow and develop a smooth supercavity.

The experimental results show that the presented design methodology of conical section shape is suitable for SVs.

\section{Nomenclature}

$c_{a}$ : Characteristic parameters of position force

$c_{x n}$ : Drag coefficient of cavitator

$c_{z}^{\beta}$ : Position derivative of lateral force coefficient

$c_{\omega}$ : Characteristic parameters of damping force

Fr: Froude number

g: Gravitational acceleration

$J: \quad$ Rotational inertia about vertical axis

$K_{r a}$ : Oscillating coefficient

$K_{\psi}$ : Ratio of yaw angle to sideslip angle

$k_{1}$ : Constant

$k_{2}$ : Constant

$L_{a}$ : Arm of lateral force about mass center

$L_{n}$ : Distance from cavitator center to mass center

$L_{n}^{\prime}$ : Distance from instantaneous velocity center to mass center 
$L_{t}$ : Length of vehicle

$L_{w}$ : Distance from stern plane center to mass center

$L_{z}$ : Conical section length

$m$ : Mass of vehicle

$M_{y}$ : Yaw moment

$P_{c}$ : Cavity pressure

$P_{v}: \quad$ Saturated water vapor pressure

Q: $\quad$ Volumetric flow rate of gas

$\bar{Q}: \quad$ Air entrainment coefficient

$R_{c}$ : Radius of supercavity

$R_{n}$ : Radius of cavitator

$R_{t}:$ Radius of cylindrical section

$R_{z}$ : Radius of conical section

$r_{z w}: z$-component of aft cavity center in body coordinate system

$S: \quad$ Characteristic area of vehicle

$S_{c}: \quad$ Spiral pitch

$t$ : Time

$v: \quad$ Sailing speed

$x_{s x}$ : Distance from instantaneous velocity center to cavitator

$Z$ : $\quad$ Lateral force

$z_{x}$ : Oscillation position at position $x$

$z_{e}: \quad z$-component of mass center in ground coordinate system

$z_{n}: \quad$ z-component of cavitator center

$z_{w}: \quad z$-component of stern plane center in ground coordinate system

$\beta: \quad$ Angle of sideslip

$\rho: \quad$ Density of fluid

$\sigma: \quad$ Cavitation number

$\sigma_{v}: \quad$ Natural cavitation number

$\sigma_{c}:$ Ventilated cavitation number

$\tau: \quad$ Time delay

$\omega$ : Angular frequency of the auto-oscillation

$\varphi_{\psi}:$ Phase of yaw angle

$\psi$ : Yaw angle

$\psi_{A q}$ : Amplitude of yaw angle

$\Psi: \quad$ Trajectory deflection angle

$\omega: \quad$ Frequency of auto-oscillation

$\mu$ : $\quad$ Relative density

\section{Subscripts}

A: Amplitude

$b$ : $\quad$ Body-fixed coordinate system

max: Maximum

$\tau: \quad$ Parameters at time $t-\tau$

$\infty$ : Mainstream value.

\section{Appendix}

Auto-Oscillation Characteristics of SVs. The components of distance $r_{y}$ and $r_{z}$ are expressed as

$$
\begin{aligned}
r_{y}= & \cos \theta\left(y_{e \tau}-y_{e}+y_{G}\right)-\sin \theta \cos \psi\left(x_{e \tau}-x_{e}\right) \\
& +\sin \theta \sin \psi\left(z_{e \tau}-z_{e}\right)+\sin \left(\psi-\psi_{\tau}\right) \sin \theta z_{r n} \\
& +\left(L_{n}+\alpha_{\tau} y_{e n}-\beta_{\tau} z_{r n}\right)\left[\cos \theta \sin \theta_{\tau} \cos \left(\psi-\psi_{\tau}\right)\right] \\
& +\left[\cos \left(\psi-\psi_{\tau}\right) \sin \theta \sin \theta_{\tau}+\cos \theta \cos \theta_{\tau}\right] y_{e n}, \\
r_{z}= & \sin \psi\left(x_{e \tau}-x_{e}\right)+\cos \psi\left(z_{e \tau}-z_{e}\right)+\cos \theta_{\tau} \sin \left(\psi-\psi_{\tau}\right) \\
& +\left(L_{n}+\alpha_{\tau} y_{e n}-\beta_{\tau} z_{r n}\right)-\sin \theta_{\tau} \sin \left(\psi-\psi_{\tau}\right) y_{e n} \\
& +\cos \left(\psi-\psi_{\tau}\right) z_{r n} .
\end{aligned}
$$

Time-Domain Analysis of Auto-Oscillation. The coefficients $\bar{C}$ and $\bar{S}$ are expressed as

$$
\begin{aligned}
& \bar{C}=\frac{J}{m L_{\alpha}}+L_{w}-\frac{\omega^{2} J}{c_{\alpha} L_{\alpha}}-\left(\frac{J}{m L_{\alpha}}-L_{n}\right) \cos \omega \tau, \\
& \bar{S}=\left(\frac{J}{m L_{\alpha}}-L_{n}\right) \sin \omega \tau+\frac{\omega c_{\omega}}{c_{\alpha}} .
\end{aligned}
$$

Length of Conical Section. The coefficients $A_{\sigma}, B_{\sigma}$, and $C_{\sigma}$ are expressed as

$$
\begin{aligned}
A_{\sigma} & =\frac{k_{1}}{c_{x n}}\left(\frac{L_{t}}{R_{n} k_{2}}\right)^{2}, \\
B_{\sigma} & =\left(\frac{L_{t}}{R_{n} k_{2}}\right)^{2}+\frac{2 k_{1} L_{t}}{R_{n} k_{2} \sqrt{c_{x n}}}, \\
C_{\sigma} & =\left[k_{1}-k_{1}\left(\frac{R_{t}}{R_{n}}\right)^{2}+\frac{2 \sqrt{c_{x n}} L_{t}}{R_{n} k_{2}}\right] .
\end{aligned}
$$

Streamlined Shape of Conical Section. The coefficients $C_{2 q}, C_{1}$, and $C_{2}$ are expressed as

$$
\begin{aligned}
& C_{2 q}=1-\frac{\sigma L_{z}}{R_{n} k_{2} \sqrt{c_{x n}}}, \\
& C_{1}=1-\frac{k_{1} \sigma}{c_{x n}}, \\
& C_{2}=1-\frac{\sigma x}{R_{n} k_{2} \sqrt{c_{x n}}} .
\end{aligned}
$$

\section{Data Availability}

The data used to support the findings of this study are available from the corresponding author upon request.

\section{Conflicts of Interest}

The authors declare that they have no conflicts of interest. 


\section{Acknowledgments}

This work was supported by the National Natural Science Foundation of China (grant nos. 51679202 and 51579209).

\section{References}

[1] G. V. Logvinovich, "Hydrodynamics of free-boundary flows," in Translated from the Russian (NASA-TT-F-658), P. Greenberg, Ed., US Department of Commerce, Washington, DC, USA, 1972.

[2] V. N. Semenenko, "Artificial supercavitation. Physics and calculation," in Proceedings of the RTO AVT Lecture Series on Supercavitating Flows, Von Karman Institute, Brussels, Belgium, 2001.

[3] V. N. Semenenko, "Dynamic processes of supercavitation and computer simulation," in Proceedings of the RTO AVT Lecture Series on Supercavitating Flows, Von Karman Institute, Brussels, Belgium, 2001.

[4] Y. N. Savchenko, "Control of supercavitation flow and stability of supercavitating motion of bodies," in Proceedings of the RTO AVT Lecture Series on Supercavitating Flows, Von Karman Institute, Brussels, Belgium, 2002.

[5] Y. N. Savchenko, "Supercavitation-problems and perspectives," in Proceedings of the 4th International Symposium on Cavitation (CAV2001), Paris, France, 2001.

[6] A. D. Vasin and E. V. Paryshev, "Immersion of a cylinder in a fluid through a cylindrical free surface," Fluid Dynamics, vol. 36, no. 2, pp. 169-177, 2001.

[7] J.-Y. Choi, M. Ruzzene, and O. A. Bauchau, "Dynamic analysis of flexible supercavitating vehicles using modal-based elements," Simulation, vol. 80, no. 11, pp. 619-633, 2004.

[8] M. Ruzzene and F. Soranna, "Impact dynamics of elastic stiffened supercavitating underwater vehicles," Journal of Vibration and Control, vol. 10, no. 2, pp. 243-267, 2004.

[9] I. N. Kirschner, N. E. Fine, J. S. Uhlman et al., Supercavitation Research and Development, Undersea Defense Technologies, Waikiki, Hawaii, 2001.

[10] I. N. Kirschner, D. C. Kring, A. W. Stokes, N. E. Fine, J. S. Uhlman, and S. Uhlman, "Control strategies for supercavitating vehicles," Journal of Vibration and Control, vol. 8, no. 2, pp. 219-242, 2002.

[11] J. Dzielski and A. Kurdila, "A benchmark control problem for supercavitating vehicles and an initial investigation of solutions," Journal of Vibration and Control, vol. 9, no. 9, pp. 791-804, 2003.

[12] F. Scorcelletti, C. L. Bottasso, and M. Ruzzene, "Multiple shooting solution of optimal control problems for timedelayed dynamic systems," Journal of Dynamic Systems, Measurement, and Control, vol. 131, Article ID 011009, 2009.

[13] M. Howe, A. Colgan, and T. Brungart, "On self-noise at the nose of a supercavitating vehicle," Journal of Sound and Vibration, vol. 322, no. 4-5, pp. 772-784, 2009.

[14] I. N. Kirschner, J. S. Uhlman, and J. Perkins, "Overview of high-speed supercavitating vehicle control," in Proceedings of the AIAA Guidance, Navigation, and Control Conference and Exhibit, Keystone, CO, USA, 2006.

[15] S. S. Ahn, M. Ruzzene, F. Scorcelletti, and C. L. Bottasso, "Configuration optimization of supercavitating underwater vehicles with maneuvering constraints," IEEE Journal of Oceanic Engineering, vol. 35, no. 3, pp. 647-662, 2010.

[16] V. Nguyen, B. Balachandran, and A. N. Varghese, "Supercavitating vehicle dynamics with non-cylindrical, non-symmetric cavities," in Proceedings of the ASME 2007
International Mechanical Engineering Congress and Exposition, pp. 265-272, American Society of Mechanical Engineers, Part A, Seattle, WA, USA, 2007.

[17] G. Lin, B. Balachandran, and E. H. Abed, "Nonlinear dynamics and bifurcations of a supercavitating vehicle," IEEE Journal of Oceanic Engineering, vol. 32, no. 4, pp. 753-761, 2007.

[18] G. Lin, B. Balachandran, and E. H. Abed, "Dynamics and control of supercavitating vehicles," Journal of Dynamic Systems, Measurement, and Control, vol. 130, no. 2, pp. 021003-21011, 2008.

[19] Y. Lv, T. Xiong, W. Yi, and J. Guan, "Robustness of supercavitating vehicles based on multistability analysis," Advances in Mathematical Physics, vol. 2017, Article ID 6894041, 13 pages, 2017.

[20] T. Xiong, Y. Lv, and W. Yi, "Analysis on multistable motion characteristics of supercavitating vehicle," Shock and Vibration, vol. 2017, Article ID 9712687, 11 pages, 2017.

[21] C. Huang, K. Luo, J. Dang, K. Qin, and D. J. Li, "Spatial kinetics model of supercavitating vehicles reflecting conic-like oscillation," Mathematical Problems in Engineering, vol. 2017, Article ID 3671618, 12 pages, 2017.

[22] M. Ruzzene, "Non-axisymmetric buckling of stiffened supercavitating shells: static and dynamic analysis," Computers \& Structures, vol. 82, no. 2-3, pp. 257-269, 2004.

[23] E. Alyanak, V. Venkayya, R. Grandhi, and R. Penmetsa, "Structural response and optimization of a supercavitating torpedo," Finite Elements in Analysis and Design, vol. 41, no. 6, pp. 563-582, 2005.

[24] E. Alyanak, R. Grandhi, and R. Penmetsa, "Optimum design of a supercavitating torpedo considering overall size, shape, and structural configuration," International Journal of Solids and Structures, vol. 43, no. 3-4, pp. 642-657, 2006.

[25] J. H. Choi, R. C. Penmetsa, and R. V. Grandhi, "Shape optimization of the cavitator for a supercavitating torpedo," Structural and Multidisciplinary Optimization, vol. 29, no. 2, pp. 159-167, 2005.

[26] J. S. Uhlman, "A note on the development of a nonlinear axisymmetric reentrant jet cavitation model," Journal of Ship Research, vol. 50, no. 3, pp. 259-267, 2006.

[27] R. Kuklinski, "Experimental studies in the control of cavitating bodies," in Proceedings of the AIAA Guidance, Navigation, and Control Conference and Exhibit, p. 6442, Keystone, CO, USA, 2006.

[28] E. Kawakami and R. E. A. Arndt, "Investigation of the behavior of ventilated supercavities," Journal of Fluids Engineering, vol. 133, no. 9, pp. 57-64, 2011.

[29] H. Mokhtarzadeh, G. Balas, and R. Arndt, "Effect of cavitator on supercavitating vehicle dynamics," IEEE Journal of Oceanic Engineering, vol. 37, no. 2, pp. 156-165, 2012.

[30] S. Park and S. H. Rhee, "Computational analysis of turbulent super-cavitating flow around a two-dimensional wedge-shaped cavitator geometry," Computers \& Fluids, vol. 70, pp. 73-85, 2012.

[31] E. F. Campana, G. Fasano, and D. Peri, "Penalty function approaches for ship multidisciplinary design optimisation (MDO)," European Journal of Industrial Engineering, vol. 6, no. 6, pp. 765-784, 2012.

[32] G. Fasano, G. Liuzzi, S. Lucidi, and F. Rinaldi, "A linesearchbased derivative-free approach for nonsmooth constrained optimization," SIAM Journal on Optimization, vol. 24, no. 3, pp. 959-992, 2014.

[33] A. Belegundu, E. Halberg, M. Yukish, and T. Simpson, "Attribute-based multidisciplinary optimization of undersea 
vehicles," in Proceedings of the 8th Symposium on Multidisciplinary Analysis and Optimization, p. 4865, Long Beach, CA, USA, 2000.

[34] C. Mcallister, T. Simpson, P. Kurtz, and M. Yukish, "Multidisciplinary design optimization testbed based on autonomous underwater vehicle design," in Proceedings of the 9th Symposium on Multidisciplinary Analysis and Optimization, p. 5630, Atlanta, GA, USA, 2002.

[35] W. L. Tian, Z. Y. Mao, F. L. Zhao, and Z. C. Zhao, "Layout optimization of two autonomous underwater vehicles for drag reduction with a combined CFD and neural network method," Complexity, vol. 2017, Article ID 5769794, 15 pages, 2017.

[36] P. S. Granville, "Geometrical characteristics of streamlined shapes," Technical Report 2962, The David W. Taylor Naval Ship Research and Development Centre, Department of Hydromechanics, Bethesda, MD, USA, 1969.

[37] D. Li, Q. Liu, K. Qin, C. Huang, K. Luo, and J. Dang, "Classical control of underwater supercavitating vehicles via variable splitting method," Ships and Offshore Structures, vol. 14, no. 7, pp. 765-776, 2018.

[38] Y. W. Zhang, Torpedo Trajectory and Trajectory Design, Northwestern Polytechnical University Press, Xi'an, China, 1st edition, 1999. 